\title{
CONTIGUOUS HYPERGEOMETRIC FUNCTIONS OF THE TYPE ${ }_{3} F_{2}(1)$
}

\author{
by W. N. BAILEY
}

(Received 19th December, 1953)

1. In a paper published recently in this Journal, Watson * obtained a reduction formula for the integral

$$
I_{n}=\int_{0}^{1} x^{\alpha}(1-x)^{\beta} \frac{d^{n}\left\{x^{\gamma}(1-x)^{\delta}\right\}}{d x^{n}} d x .
$$

He showed that this reduction formula could be written in the form :

If

$$
H_{n}=\sum_{r=0}^{n}(-1)^{n-r}\left(\begin{array}{l}
n \\
r
\end{array}\right)(-\gamma)_{n-r}(\beta+\delta-n+1)_{n-r}(-\delta)_{r}(\alpha+\gamma-n+1)_{r},
$$

.then

$$
(2 \sigma-n)(\sigma-n) H_{n+2}-Q_{n} H_{n+1}+S_{n} H_{n}=0,
$$

where

$$
\begin{aligned}
& S_{n}=(n+1)(\sigma-n-1)(\alpha+\beta-n)(\gamma+\delta-n)(\alpha+\gamma-n)(\beta+\delta-n), \\
& Q_{n}=(2 \sigma-2 n-1)\left\{(\beta \gamma-\alpha \delta)(\sigma+\dot{1})+\theta_{3}(n+1)(2 \sigma-n)\right\},
\end{aligned}
$$

and $2 \sigma=\alpha+\beta+\gamma+\delta, 2 \theta_{3}=\alpha-\beta-\gamma+\delta$.

As Watson remarked, $H_{n}$ can be expressed in terms of a hypergeometric series ${ }_{3} \mathrm{~F}_{2}$ with final element unity, and so (1.1) gives a relation between three contiguous hypergeometric series of this type. In fact,

Thus, if we write

$$
H_{n}=(-\gamma)_{n}(-\beta-\delta)_{n 3} F_{2}\left[\begin{array}{c}
-n,-\delta, \alpha+\gamma-n+1 ; \\
1+\gamma-n,-\beta-\delta
\end{array}\right] \text {. }
$$

$$
K_{n}={ }_{3} F_{2}\left[\begin{array}{c}
-n,-\delta, \alpha+\gamma-n+1 \\
1+\gamma-n,-\beta-\delta
\end{array}\right]
$$

the relation (1.1) becomes

$$
\begin{array}{r}
(2 \sigma-n)(\sigma-n)(\gamma-n)(\gamma-n-1)(\beta+\delta-n)(\beta+\delta-n-1) K_{n+2} \\
-Q_{n}(\gamma-n)(\beta+\delta-n) K_{n+1}+S_{n} K_{n}=0 .
\end{array}
$$

We now use the transformation $\dagger$

$$
{ }_{3} F_{2}\left[\begin{array}{c}
a, b, c ; \\
d, e
\end{array}\right]=\frac{\Gamma(d+e-a-b-c) \Gamma(d)}{\Gamma(d-a) \Gamma(d+e-b-c)}{ }_{3} F_{2}\left[\begin{array}{c}
a, e-b, e-c ; \\
e, d+e-b-c
\end{array}\right]
$$

and we find that

where

$$
\begin{aligned}
& K_{n}=\frac{(-1)^{n}(-\alpha-\beta)_{n}}{(-\gamma)_{n}} L_{n}, \\
& L_{n}={ }_{3} F_{2}\left[\begin{array}{c}
-n,-\beta, n-2 \sigma-1 ; \\
-\beta-\delta,-\alpha-\beta
\end{array}\right] .
\end{aligned}
$$

* Watson (3). I am indebted to Professor Watson for showing me his paper before publication.

$\dagger$ See Bailey (1), 18. 
Thus (1.2) becomes

$$
\begin{aligned}
& (2 \sigma-n)(\sigma-n)(\beta+\delta-n-1)(\alpha+\beta-n-1) L_{n+2}+Q_{n} L_{n+1} \\
& +(n+1)(\sigma-n-1)(\gamma+\delta-n)(\alpha+\gamma-n) L_{n}=0
\end{aligned}
$$

This relation is of a simpler kind than (1.2), since only two of the parameters in $L_{n}$ depend on $n$.

In this paper I show how to obtain relations between contiguous hypergeometric functions of the type ${ }_{3} F_{2}$. We shall incidentally obtain another proof of Watson's reduction formula, and we shall see that (1.2) and (1.4) are true when $n$ is not restricted to be a positive integer.

2. We denote by $F$ the function

$$
{ }_{3} F_{2}\left[\begin{array}{c}
a, b, c ; x \\
d, e
\end{array}\right]
$$

and by $F_{a+}, F_{a-}$ the same function when $a$ is changed to $a+1, a-1$, with a similar notation when the other parameters are so changed.

It is easily shown that, if $\delta$ denotes $x d / d x$,

$$
a F_{a+}=(\delta+a) F .
$$

Also, from the differential equation

$$
[\delta(\delta+d-1)(\delta+e-1)-x(\delta+a)(\delta+b)(\delta+c)] F=0,
$$

we get, changing $a$ into $a-1$,

$$
\begin{aligned}
(a-1)(d-a)(e-a) F_{a-} & \\
= & {\left[\left\{\delta^{2}+\delta(d+e-a-1)+(d-a)(e-a)\right\}-x(\delta+b)(\delta+c)\right](\delta+a-1) F_{a_{-}}, }
\end{aligned}
$$

and so, by $(2.1)$,

$$
(d-a)(e-a) F_{a-}=\left[\left\{\delta^{2}+\delta(d+e-a-1)+(d-a)(e-a)\right\}-x(\delta+b)(\delta+c)\right] F .
$$

Similarly we can show that *

$$
(e-1) F_{e-}=(\delta+e-1) F,
$$

$$
\begin{aligned}
& x(a-e)(b-e)(c-e) F_{e+} \\
& \quad=e\left[\delta(\delta+d-1)-x\left\{\delta^{2}+\delta(a+b+c-e)+b c+c a+a b-e(a+b+c)+e^{2}\right\}\right] F^{\prime} .
\end{aligned}
$$

By repeated applications of these formulae, as I remarked in (2), the function

$$
{ }_{3} F_{2}\left[\begin{array}{c}
a+l, b+m, c+n ; x \\
d+p, e+q
\end{array}\right]
$$

where $l, m, n, p, q$ are integers, can be expressed in terms of $F, \delta F, \delta^{2} F$, and from four relations of this type we can eliminate $F, \delta F, \delta^{2} F$ and so obtain a homogeneous linear relation between any four contiguous functions.

Now, when $x=1$, the relation (2.2) simplifies and gives $\delta^{2} F$ in terms of $\dot{F}$ and $\delta F$, and by operating on (2.2) with $\delta, \delta^{2}, \ldots$, we can find $\delta^{2} F, \delta^{3} F, \ldots$ in terms of $F$ and $\delta F$. Thus the function (2.6), with $x=1$, can be expressed in terms of $F$ and $\delta F$, and so there is a homogeneous linear relation connecting any three contiguous functions of the type ${ }_{3} \mathrm{~F}_{2}(1)$.

* Compare Bailey (2), $\S 4$, where (2.5) is given incorrectly. 
3. I now apply this method to prove (1.4) when $n$ is not restricted to be an integer. From (2.3), with $b$ changed to $b+1$, we get

$$
\begin{aligned}
& b(d-a)(e-a) \cdot F_{a-, b+} \\
& \quad=\left[\left\{\delta^{2}+\delta(d+e-a-1)+(d-a)(e-a)\right\}-x(\delta+b+1)(\delta+c) \cdot\right](\delta+b) F .
\end{aligned}
$$

Now put $x=1$ in (2.2) and (3.1), and we get

and

$$
\left[\delta^{2}(d+e-a-b-c-2)+\delta\{(d-1)(e-1)-b c-c a-a b\}-a b c\right] F=0
$$

$$
\begin{aligned}
b(d-a)(e-a) F_{a-, b+} & =[\delta(d+e-a-b-c-2)+(d-a)(e-a)-c(b+1)](\delta+b) F \\
& =[\delta(b-a+1)(d+e-a-b-c-1)+b(d-a)(e-a)-b c(b-a+1)] F
\end{aligned}
$$

on using (3.2). Now interchange $a$ and $b$ in (3.3), and eliminate $\delta F$ from the two relations, and we get

$$
\begin{aligned}
& b(a-b+1)(d-a)(e-a) F_{c-, b+}-a(b-a+1)(d-b)(e-b) F_{a+, b-} \\
& =(a-b)[c(b-a+1)(a-b+1)+d e(a+b-1)-2(d+e) a b+a b(a+b+1)] F \ldots
\end{aligned}
$$

This is equivalent to (1.4) when $n$ is not necessarily an integer, and (1.2) can be derived from it by using (1.3). A direct proof of (1.2) could be given by using the method of this paper, but, although $S_{n}$ is easily obtained, the derivation of the form of $Q_{n}$ given by Watson entails extremely involved work. On the other hand, the derivation of the value of $Q_{n}$ when obtaining (1.4) from (3.4) is quite brief.

It will be noticed that, when $d+e=a+b+c+1$, that is, when the ${ }_{3} F_{2}$ are Saalschützian (though not necessarily terminating), (3.3) gives a simple relation between $F_{a-, b+}$ and $F$.

The formula (3.4) is a degenerate form of a relation connecting four functions of the type ${ }_{3} F_{2}(x)$. Thus, from $(2.2)$ and (3.1) it is easily shown as above that, when the final element of each ${ }_{3} F_{2}$ is $x$,

$$
\begin{array}{r}
b(a-b+1)(d-a)(e-a) F_{a-, b+}-a(b-a+1)(d-b)(e-b) F_{a+, b-} \\
=(a-b)[c(b-a+1)(a-b+1) x+d e(a+b-1)-2(d+e) a b+a b(a+b+1)] F \\
+(a-b+1)(a-b)(a-b-1)(1-x) \delta F
\end{array}
$$

where, of course, $\delta F=\frac{a b c x}{d e} F_{a+, b+, c+, d+, e+}$.

4. By using the formula*

$$
{ }_{3} F_{2}\left[\begin{array}{c}
a, b, c ; \\
d, e
\end{array}\right]=\frac{\Gamma(d) \Gamma(e) \Gamma(s)}{\Gamma(c) \Gamma(s+a) \Gamma(s+b)}{ }_{3} F_{2}\left[\begin{array}{c}
d-c, e-c, s ; \\
s+a, s+b
\end{array}\right]
$$

where $s=d+e-a-b-c$, to transform the three series in (3.4), we can derive a relation equivalent to

$$
\begin{aligned}
d(d-1)(e-d-1)(a-e)(b-e)(c-e) F_{d-, e+}-e(e-1)(d-e-1)(a-d)(b-d)(c-d) F_{d+, e-} \\
=d e(d-e)[(d+e-1)(b c+c a+a b)-(2 d e-d-e+1)(a+b+c) \\
+(d+e)(d-1)(e-1)+d e-2 a b c] F . \ldots \ldots \ldots \ldots . . . .
\end{aligned}
$$

* See Bailey (1), § 3.2. 
This can also be easily derived directly by the method of this paper. Perhaps it is worth mentioning the formulae

$$
\begin{aligned}
& a(d+e-a-b-c-1) F_{a+}-(d-a)(e-a) F_{a-} \\
& =[a(2 d+2 e-2 a-b-c-1)-d e+b c] F, \\
& e(e-1)(d+e-a-b-c-1) F_{e-}-(a-e)(b-e)(c-e) F_{e+} \\
& =e\left[(e-1)(d+e-1)-(a+b+c)(2 e-1)+b c+c a+a b+e^{2}\right] F, \\
& d(d-1)(e-a) F_{a-, d-}-a(b-d)(c-d) F_{a+, d+} \\
& =d[(d-1)(e-a)-b c+c a+a b-a d] F^{\prime},
\end{aligned}
$$

the last of which can be derived from (4.3) by using (4.1).

From these results further relations can be found by transforming the series by means of (1.3) and (4.1). It would be a very tedious process to obtain some of these results directly by the general method of this paper.

5. Similar results are true for series of any order. For example, for the function

$$
{ }_{4} F_{3}\left[\begin{array}{c}
a, b, c, d ; x \\
e, f, g
\end{array}\right]
$$

the following results are true :

(i) There is a homogeneous linear relation between any five contiguous functions.

(ii) When $x=1$, there is a homogeneous linear relation between any four contiguous functions.

(iii) When $x=1$ and the parameters are such that $\Sigma e-\Sigma a=2$, there is a homogeneous linear relation connecting $F_{a+, b-}, F$ and $F_{a-, b+}$.

\section{REFERENCES}

(1) W. N. Bailey, Generalized Hypergeometric Series (Cambridge, 1935). $115-18$.

(2) W. N. Bailey, " Associated Hypergeometric Series ", Quart. J. of Math. (Oxford), 8 (1937),

(3) G. N. Watson, "A Reduction Formula", J. Glasgow Math. Ass., 2 (1954), 57-61.

\section{Bedford College}

REGENT'S PARK

LONDON, N.W.1 